\title{
Principios y conclusiones en una axiomatización de la astronomía de Acerca del cielo I de Aristóteles
}

Manuel Berrón ${ }^{*}$

\begin{abstract}
Resumen: En Acerca del cielo I se encuentran cuatro pruebas fundamentales de la cosmología aristotélica, a saber: la prueba de la existencia del éter, la prueba de la finitud del universo, la prueba de su unicidad y la prueba de su ingenerabilidad e incorruptibilidad. Ofrecemos una reconstrucción parcial del conjunto de estas cuatro pruebas para poner en evidencia que ellas utilizan un grupo semejante de premisas y definiciones. En efecto, nuestro propósito es mostrar que dicho conjunto de pruebas utiliza los mismos principios y, de este modo, que en una reconstrucción axiomática del total de pruebas podemos hallar un número semejante, o incluso mayor, de premisas que de conclusiones. Así, encontramos una forma alternativa para una axiomatización posible en Acerca del cielo de Aristóteles. Palabras clave: Aristóteles, Acerca del cielo, demostración científica, axiomatización.
\end{abstract}

\begin{abstract}
In On The Heavens there are four crucial proofs of Aristotelian cosmology, namely: proof of the aether existence, proof of the finitude of the universe, proof of its unicity and proof of its ungenerability and its indestructibility. We do a partial reconstruction of the hole of these four proofs to show that they use a similar set of premises and definitions. In fact, our goal is to show that such set of proofs uses the same principles and, in this way, that in a axiomatic reconstruction of the hole of proofs we can find a similar number, even higher, of premises than conclusions. Thus, we find an alternative way for a possible axiomatization on Aristotle's On The Heavens.
\end{abstract}

Key words: Aristotle, On The Heavens, scientific demonstration, axiomatization.

\footnotetext{
* Doctor en Humanidades, mención Filosofía, por la Universidad Nacional de Rosario. Profesor en la Facultad de Humanidades y Ciencias y en la Facultad de Arquitectura de la Universidad Nacional del Litoral. Ha sido becario postdoctoral del CONICET. Dirección electrónica: manuel.berron@gmail.com.
} 


\section{Introducción}

Abordaremos la presentación final que adquiere Acerca del Cielo I (DC I) atendiendo al modo en que son elaboradas las demostraciones sobre los tópicos astronómicos en cuestión. Nuestra línea de lectura adhiere a la opinión menos difundida de que Aristóteles, en sus textos científicos tales como Acerca del cielo, Meteorológicos, Acerca de la generación y la corrupción, entre los más destacados, está siguiendo sus prescripciones metodológicas expuestas principalmente en los Analíticos. Entre estas prescripciones, nos interesa destacar que es factible encontrar en tales textos el uso de la demostración científica y, además, que tales demostraciones soportan el formato del silogismo. ${ }^{1}$ En efecto, asumimos un silogicismo moderado ${ }^{2}$ puesto que suponemos que las demostraciones, si bien no están expresadas en el formato del silogismo, son capaces de admitirlo. En ese mismo sentido, además de mostrar la admisibilidad para las demostraciones del formato del silogismo, pretendemos exhibir que entre las premisas primeras de éstos

${ }^{1}$ Tal es la línea sugerida primeramente por A. Gotthelf en "First principles in Aristotle's Parts of Animals”, editado por A. Gotthelf y J. G. Lennox en Philosophical Issues in Aristotle's Biology, Cambridge, 1987, y en "The elephant's nose: Further reflections on the axiomatic structure of biological explanation in Aristotle", editado por Kullmann y Föllinger en Aristotelische Biologie, Intentionen, Methoden, Ergebnisse, Stuttgart, 1997. Esta línea fue continuada y desarrollada por W. Detel Aristoteles Analytica Posteriora, Akademie Verlag, 1993, y "Why all animals have a stomach”, en Kullman y Föllinger (Ed.), op. cit., 1997.

${ }^{2}$ El término lo tomamos de Ferejohn (Ferejhon, Michael, The Origins of Aristotelian Science, New Haven/ Londres, Yale University Press, 1991), quien distingue entre silogicistas y antisilogicistas. Los segundos son aquellos que defienden la tesis de la discrepancia (la línea de crítica de Solmsen continuada por Barnes, cfr. Solmsen, Friedrich, Die Entwicklung der aristotelischen Logic und Rhetorik, Berlin, Weidmann, 1929 y Barnes, Jonathan, “Aristotle's Theory of Demonstration", en Phronesis 14) mientras que los primeros la rechazan. Los silogicistas imprimen un carácter geometricista a la estructura de las demostraciones científicas llevando la lectura más allá de lo que afirma el propio Aristóteles (la "Introduction" de Ross en Aristotle, Prior and Posterior Analytics, Oxford, Oxford University Press, Trad. D. Ross, 1949 y Hintikka, Jakko, "On the Ingredients of an Aristotelian Science", en Nous VI - 1, 1972). Ferejohn se separa de ambos extremos para defender un silogicismo en el sentido de que las demostraciones científicas toman el formato del silogismo, pero sin el agregado de la lectura geometrizante (cf. Ferejohn: op. cit., 18-9). 
se encuentran principios científicos. Claramente, sólo tendremos auténticas demostraciones científicas si los puntos de partida son principios y, en esa dirección, pretendemos exhibir que, efectivamente, entre dichas premisas encontramos definiciones científicas, $i$. e., los genuinos principios de la ciencia según los Analíticos.

Nuestro enfoque se contrapone a la línea interpretativa más difundida en la segunda mitad del S. XX que hizo hincapié en la aparente contradicción o discrepancia entre la filosofía de la ciencia de Aristóteles presentada en los Analíticos y su práctica científica. ${ }^{3}$ La tesis emblemática de la discrepancia articuló armónicamente con la poderosa relectura de la dialéctica aristotélica (especialmente a partir del III Symposium Aristotelicum de 1963 dedicado a la dialéctica y, con ella, a Tópicos) que ubicó a ésta como herramienta metodológica predilecta. ${ }^{4}$ La dialéctica, así revalorizada, se constituyó en la principal herramienta tanto en la heurística de los principios como en la justificación de los mismos y, en este marco, las herramientas propuestas por Aristóteles como claves en el desarrollo del conocimiento científico -principalmente la demostración científica y la inducción- pasaron a ocupar un lugar secundario o incluso inexistente en la práctica científica aristotélica.

Con todo, el veredicto extremo al que se arribó comenzó a ser relativizado al profundizarse en el estudio de las obras de ciencia natural de Aristóteles. Este hecho aconteció especialmente a partir de la década de 1980 con el desarrollo de la investigación sobre las obras de biología del estagirita. ${ }^{5}$ En ese marco, cuyo amplio universo de tópicos a discutir no podemos

${ }^{3}$ El autor emblemático de esta interpretación es J. Barnes quien soluciona el problema de la discrepancia proponiendo que los textos de ciencia están elaborados en vistas de descubrir los principios mientras que la teoría de la demostración sólo es útil para la exposición a partir de principios ya conocidos (cf. Barnes, op. cit.,138). ${ }^{4}$ El organizador del Simposio, G. E. L. Owen, fue uno de los más fuertes impulsores de la reivindicación de la dialéctica como herramienta central a partir de su influyente artículo "Tithenai ta phainómena" presentado en el II Simposio (editado en Susan Mansion, Aristote et les problemes de méthode, Louvain-la-neuve, Editions de L'Institut supérieur de philosophie, 1980). También vieron la luz dos influyentes obras en este período: de Wolfgang Wieland, Die aristotelische Physik, Vandenhoeck \& Ruprecht. Göttingen, 1962 y de Pierre Aubenque, Le problème de l'etre chez. Aristote, Essai sur la problematique aristotélicienne, Paris, 1962.

${ }^{5}$ Se destacan las compilaciones realizadas por Lennox y Gotthelf (de 1987, ya citada) y de L. Judson (Lindsay Judson (ed.), Aristotle's Physics, A collection of Essays, 
abordar dadas las dimensiones de un trabajo de este tipo, cabe señalarse que se revalorizó el aporte de la investigación empírica en desmedro de un uso exclusivo de la dialéctica ${ }^{6}$ así como también se volvió a ponderar el uso de la demostración científica o, al menos, de la existencia de una trama demostrativa subyacente en las obras de biología. ${ }^{7}$ Bastan éstos como ejemplos de algunos de los más importantes temas reconsiderados en los últimos años. No pretendemos profundizar en ello sino que, centrándonos en nuestro trabajo, el objetivo que perseguimos particularmente consiste, por un lado, en volver manifiesto que las diferentes demostraciones realizadas por Aristóteles sobre los tópicos mencionados en DC I, a saber: la existencia del éter, la finitud, la ingenerabilidad e incorruptibilidad y la unicidad del universo, admiten el formato del silogismo y, además, utilizan un mismo "soporte teórico". ${ }^{8}$ Tal soporte se encuentra constituido por un conjunto de definiciones brindadas por Aristóteles en el comienzo de $D C$ que, luego y en la medida que el asunto discutido lo requiere, se ve ampliado con nuevas definiciones. Por otro lado, una vez que hayamos logrado la reconstrucción y mostremos que las pruebas se remiten a un mismo soporte teórico, estaremos en condiciones de valorar qué relación existe entre las premisas iniciales y las conclusiones probadas, i. e., entre los principios y las conclusiones. En efecto, podremos valorar qué número de premisas son requeridas para la construcción de las distintas demostraciones. Finalmente, esta reconstrucción de las pruebas nos permitirá visualizar cuál es el modo en que se puede comprender una axiomatización ${ }^{9}$ de la astronomía presentada en DC I.

Oxford, 1991) y que reúnen los trabajos de las principales investigaciones sobre biología y física en Aristóteles de dicha década.

${ }^{6}$ Cf. Bolton, Robert, "Aristotle's Method in Natural Science, Physics I", en L. Judson, op. cit., pp. 1-30.

${ }^{7}$ Cf. Gotthelf, A., "First principles in Aristotle's Parts of Animals".

8 La expresión "soporte teórico" es nuestra opción para traducir el término inglés background utilizado por W. Detel para aludir al conjunto de principios que constituyen el trasfondo teórico sobre el que Aristóteles construye sus demostraciones (cf. Detel, W., "Why all animals have stomach", p. 87 ss.). He contribuido en el desarrollo de esta noción con un trabajo que se encuentra en prensa (cf. Berrón, Manuel, "El soporte teórico subyacente en las pruebas de la unicidad del cielo en Acerca del cielo I 8 de Aristóteles", en Teorema, Universidad de Oviedo, 2015).

${ }^{9}$ Entiéndase por “axiomatización” simplemente la posibilidad de distinguir en el marco de una disciplina científica entre proposiciones básicas y subordinadas, i. e., 
Para alcanzar dicho fin, tomaremos pasajes puntuales de $D C$ I en donde encontramos demostraciones claves que podemos reconstruir, como hemos señalado, bajo el formato del silogismo. Luego de su reconstrucción, podremos mostrar el modo en que tales demostraciones se conectan con las hipótesis iniciales -tal es el modo en que Aristóteles las llama- expresadas en el formato de definiciones. De este modo, en (1) haremos una desarrollo de aspectos relevantes de la discusión actual sobre el tópico; en (2) explicitaremos cuáles son las hipótesis o puntos de partida sobre los que se construyen las demostraciones; a continuación, por medio de una selección de pasajes de DC I, reconstruiremos distintas demostraciones: (2.1) hipótesis o puntos de partida; (2.2) prueba de la existencia del éter; (2.3) prueba de la finitud del universo; (2.4) prueba de la unicidad del universo; y (2.5) prueba de la ingenerabilidad e incorruptibilidad del universo. En (3) conectaremos estas demostraciones con las definiciones presentadas en (2). En (4) discutiremos el alcance del examen de los textos y (5) extraeremos algunas conclusiones.

\section{Principales aspectos de la discusión}

Me detendré en tres tópicos centrales referidos a la revalorización de la propuesta epistemológica presentada en los Analíticos y el modo en que estos aspectos del problema pueden ser encontrados en las obras de ciencia natural. ${ }^{10}$ Para ello, tocaremos brevemente aquí los siguientes temas tal como han sido abordados recientemente por distintos autores: (a) el uso implícito de la demostración científica; (b) el uso de definiciones como principios de las ciencias; (c) el modelo posible para una axiomática alternativa.

(a) Como he señalado, la visión preponderante en la segunda mitad del S. XX sobre el tópico de la demostración científica asume que ésta no habría sido utilizada realmente en los tratados de ciencia natural. Es indiscutible que la presentación final de las obras aristotélicas posee un formato

principios y teoremas. Sobre sistemas axiomáticos, v. Cassini, Alejandro, El juego de los principios, Buenos Aires, AZ, 2006.

${ }^{10} \mathrm{Me}$ limito a referirme a autores que considero pueden ser valiosos en mi propuesta de conciliar lineamientos metodológicos de los Analíticos con las obras de ciencia natural. Razones de espacio me fuerzan a no profundizar en los autores que critican esta posibilidad. Con todo, estos últimos son los que más reconocimiento tienen en la bibliografía crítica; ocasionalmente me referiré a ellos. 
particular: escasas referencias empíricas (con la honrosa excepción de las obras de biología), numerosos argumentos dialécticos, críticas a otros filósofos, argumentos que apelan a la geometría, etc. Con todo, tildar a Aristóteles de contradictorio- tal es la perspectiva de la tesis de la "discrepancia" -parece ser simplificador. En una dirección diferente plantea las cosas M. Ferejohn, quien propone distintas etapas en la exposición aristotélica. En una primera etapa "presilogística" abundaría un "eclecticismo metodológico" en el sentido de que no hay un sólo método para acceder a los principios sino que Aristóteles articularía los distintos métodos por él investigados, a saber: la dialéctica y sus herramientas, el aporte de la investigación empírica e, incluso, la articulación de éstos con la demostración científica. Tal metodología híbrida recurriría, de modo preponderante pero no exclusivamente, a la dialéctica ${ }^{11}$ mientras que, en otra etapa, de corte demostrativo se apelaría a la demostración científica y, con ella, al recurso del silogismo. Ferejohn convierte el problema planteado por la aparente contradicción en un nuevo acertijo: ¿cómo conciliar los distintos métodos caracterizados por Aristóteles a lo largo del Organon en su uso particular en los tratados de ciencia? ${ }^{12}$ De este modo, la lectura de Ferejohn genera un espacio para pensar en la articulación de la demostración científica en los tratados científicos y deja planteada la posibilidad de que ella opere demostrativamente a partir de principios. Quien ha hecho un aporte relevante en esta dirección es A. Gotthelf puesto que asume que es posible concebir una estructura axiomática implícita en Partes de los animales y, con ella, un uso implícito de la demostración científica. ${ }^{13}$ Gotthelf establece la existencia de estructuras

${ }^{11}$ A ciencia cierta, Ferejohn sostiene que esta etapa recurre a la división platónica (cf. Ferejohn, M., op. cit., p. 6), pero consideramos que este estadio es más complejo y que integra la dialéctica en sentido aristotélico -como es presentada en Tópicos- y, por ello, que tiene a disposición la división, pero también otros recursos.

${ }^{12}$ A ciencia cierta, Ferejohn se ocupa de examinar el modo en que la división (APo. II 13), de raigambre platónica pero en versión aristotélica, puede ser útil en el hallazgo de las definiciones, i. e., de los principios de las ciencias (cf. Ferejohn, M., op. cit., pp. 19 ss.). Es nuestra lectura la que aumenta al conjunto del Organon el enfoque que convierte el problema de la discrepancia en el problema de la conciliación de métodos.

${ }^{13}$ Cf. Gotthelf, A., op. cit. p. 169. Además, Kullmann y Lennox mostraron también el uso de la apódeixis en HA I 6 (cf. Kullmann, W., Wissenschaft und Methode. Berlin: Walter de Gruyter. 1974: p. 81 n. 8 y Lennox, James, "Divide and explain: 
argumentativas en $P A$ que claramente exponen la existencia de presupuestos teóricos utilizados demostrativamente a lo largo de PA II-IV. Su hipótesis de lectura es que existe un trasfondo teórico común que opera como soporte para el grueso de las argumentaciones presentadas en esta obra. La estructura axiomática sólo aparecería si se hiciera el esfuerzo por mostrar el modo en que las diversas pruebas presentadas se ponen explícitamente en conexión con dicho trasfondo. De este modo, la tesis de Gotthelf es que la demostración científica está siendo utilizada realmente en los tratados de biología pero no explícitamente. Dado que para que haya una demostración es preciso contar con principios a partir de los cuales construirla, si se logra mostrar cuáles son los principios utilizados y el modo en que ellos actúan en contextos demostrativos se podría afirmar que nos encontramos con verdaderas apodeixis, las que, incluso, estarían cumpliendo con los requerimientos de APo. I 2 71b21-22. W. Detel retoma esta idea ampliando el alcance central de la tesis: en efecto, él sostiene que la teoría subyacente, que llamo aquí "soporte teórico", no se limita sólo a la disciplina en cuestión sino que se ramifica en todas aquellas disciplinas que pueden aportar principios explicativos causales. ${ }^{14}$ Detel defiende también el uso implícito de la demostración científica pero potencia la tesis al ramificar el uso de la demostración en múltiples direcciones. El resultado es un soporte teórico en formato de red teórica transdisciplinar, ${ }^{15}$ aunque organizada jerárquicamente, que permite brindar múltiples explicaciones para la conclusión examinada (Detel denomina a esta búsqueda como el trabajo del análisis $\left.{ }^{16}\right)$.

(b) Un importante y reciente esfuerzo por reconsiderar la importancia de las definiciones como principios de las ciencias y su conexión con la teoría de la demostración puede ser encontrado en el rico examen del asunto realizado por D. Charles. ${ }^{17}$ Entre los numerosos aspectos de su trabajo, nos interesa destacar la trabazón que urde entre la demostración y

the Posterior Analytics in practice", en Gotthelf y Lennox, op. cit., pp. 101-2).

${ }^{14}$ Detel, W.: "Why all animals have stomach", pp. 83-4 y Detel, W.: "Aristotle's Logic and Theory of Science", en Gill y Pellegrin (Eds.), A Companion to Ancient Philosophy, Blackwell, 2006, p. 246.

${ }^{15}$ Con "transdisciplinar" queremos decir que, en el marco de una prueba específica, es lícito recurrir a principios de distintas ciencias sin caer el error del traspaso entre géneros diferentes.

${ }^{16}$ Cf. Detel, W., op. cit., pp. 245-7.

${ }^{17}$ Charles, D., Aristotle on Meaning and Essence. Oxford, OUP, 2000. 
la definición. Su lectura de APo. II 8-10 lo conduce a sostener que, según Aristóteles, sólo nos encontramos con una genuina definición cuando la podemos utilizar en un contexto demostrativo. La crítica de Aristóteles al método de la deducción general -como Charles la denomina- y de la división en $A$ Po. II 4-6 consiste en observar que tales recursos permiten hallar definiciones, pero que resultan impotentes para lograr certeza sobre si tales definiciones son correctas. ${ }^{18}$ Ambos métodos no sobrepasan lo que él llama la Restricción definicional puesto que no son capaces de brindar razones que aseguren que la definición es correcta. ${ }^{19}$ Además, parte de la Restricción definicional consiste en una Condición de prioridad (también llamada Condición de simplicidad ${ }^{20}$ ). Esta exigencia supone para el método que alcanza la esencia que debe también legitimar la afirmación de que alguna característica presente en $\mathrm{X}$ es prioritaria respecto de toda otra característica de $\mathrm{X} .{ }^{21}$ Hechas estas críticas a la deducción general y a la división, Aristóteles propondría a la demostración como el único método capaz de aportar razones fehacientes para garantizar haber hallado genuinas definiciones. Según su interpretación, los términos que componen una definición deben proveer la información causal, de modo que al usar las definiciones en contextos demostrativos ellos introduzcan alguna de las cuatro causas en la explicación del fenómeno. Así, la explicación, es decir, la construcción de una demostración científica, captura el "orden causal" que conecta el fenómeno a explicar con las características relevantes y necesarias presentes en la definición. ${ }^{22}$ De este modo, encontramos que la lectura presentada por Charles tiene puntos de contacto con los lineamientos generales desarrollados en (a) de Gotthelf y Detel. En efecto, estos dos autores aceptan el uso de la demostración científica en los tratados de ciencia mientras que Charles construye una interpretación en la que vincula indisolublemente la práctica demostrativa -tal como debería ser hallada en los tratados de ciencia- con el contexto de prueba de las definiciones. Ambas hipótesis pueden ser conectadas si somos capaces de mostrar que en un texto científico específico tal como $D C$ podemos encontrar tanto demostraciones

\footnotetext{
${ }^{18}$ Charles, D., op. cit., pp. 196-200.

${ }^{19}$ Ibid. p. 189.

${ }^{20}$ Ibid. p. 195.

${ }^{21}$ Ibid. p. 191.

${ }^{22}$ Ibid. p. 200.
} 
como definiciones. De hecho, sólo obtendremos verdaderas definiciones si las tenemos operando como principios de las demostraciones a la vez que sólo obtendremos genuinas demostraciones si en ellas actúan definiciones como principios. ${ }^{23}$

(c) En su distinción entre silogicistas y antisilogicistas, Ferejohn destaca que su propia lectura se encuentra entre los primeros. Sin embargo, señala que los silogicistas más extremos asocian a la lógica del silogismo de $A P r$. con el fundacionismo presentado en $A P o$. I 1-3 pero bajo una perspectiva geometrizante (y así proto-euclídea). ${ }^{24}$ Este enfoque se asociaría a la visión euclidiana de un todo demostrativo completo con la conocida relación entre pocos primeros principios y sus proposiciones lógicamente derivadas en un número mayor. Sin embargo, Ferejohn señala que tal enfoque es demasiado radical y que no acuerda con distintas afirmaciones aristotélicas. Por ello, concluye que hay que pensar en una concepción fundacionista pre-euclídea, anterior en el tiempo y distinta de la atribuida a Euclides, para interpretar APo. I 1-3, es decir, una concepción que integra el silogismo como herramienta lógica que introduce el vector causal pero que no por ello adquiere, en su presentación del conjunto de la demostraciones, el aspecto de una ciencia axiomatizada en sentido euclídeo. ${ }^{25}$ Detel aporta interesantes elementos a favor de esta lectura disonante al señalar que, para una conclusión particular, pueden construirse numerosas pruebas distintas. En efecto, su interpretación del soporte teórico como una base teórica transdisciplinar muestra que las cadenas argumentativas antes que remontarse a pocos primeros principios se ramifican en múltiples direcciones, tantas como lo permita la materia del asunto. ${ }^{26}$ Una ciencia axiomatizada en este sentido se aleja de aquella interpretación estándar presentada, por ejemplo,

${ }^{23}$ Esta aparente circularidad no debe, con todo, entenderse como una dificultad para probar las definiciones. No debe perderse de vista que Aristóteles elude el trilema de la fundamentación señalando que sobre los principios tenemos otro tipo de conocimiento. La respuesta clásica puso allí a la inducción, que culmina en un insight de los principios (cf. Ross, D., op. cit., p. 49), y la ortodoxia de los años '60 a la dialéctica. Tentativamente, hoy podemos brindar una respuesta alternativa a dicho dilema.

${ }^{24}$ Esta es la lectura estándar de Aristóteles como el primero en proponer un tipo de axiomatización (cf. Cassini, A., op. cit., pp. 19 ss).

${ }^{25}$ Ferejohn, M., op. cit. pp. 17-8.

${ }^{26}$ Detel, W., "Why all animals have stomach", p. 83. 
por Barnes, ${ }^{27}$ especialmente en la restricción referida al número de los principios. Tanto la interpretación de Ferejohn como la de Detel permiten concebir una axiomática alternativa donde el número de las conclusiones es semejante o incluso menor que el de las premisas. Desarrollaremos este aspecto de la cuestión en (4), cuando extraigamos conclusiones de lo trabajado en (2) - (3).

Hasta aquí con algunos importantes aportes referidos a aspectos relevantes de la crítica erudita contemporánea que son pertinentes al tema en cuestión. Volveremos sobre ellos luego del examen de la base textual.

\subsection{Las hipótesis: los puntos de partida}

Tomaremos como puntos de partida a las llamadas definiciones presentadas en el inicio de DC I 1-2. Tales definiciones constituyen lo que llamamos el "soporte teórico". Como ya hemos anticipado, consideramos por tal aquí a las definiciones utilizadas como principios de las ciencias. ${ }^{28}$ Aristóteles presenta un complejo conjunto de definiciones en el inicio mismo de DCI con la clara finalidad de utilizarlas para desarrollar sus demostraciones. ${ }^{29}$ Tales definiciones, que Aristóteles llama bypothésthai, supuestas, (DC I 3 269b20) o directamente hypóthesis, hipótesis, (DC I 8276b8), son el punto de partida del tratado y pueden sintetizarse en este conjunto:

${ }^{27}$ Cf. Barnes, J.: op. cit. He realizado críticas puntuales a la presentación realizada por Barnes en Berrón, M.: "Claves para una lectura alternativa de la axiomática en Aristóteles: el caso de Acerca del cielo I", en Ideas y valores, Revista Colombiana de Filosofía, Vol. 159, Diciembre 2015.

${ }^{28}$ Conviene tener en cuenta que no discutimos en este espacio cómo se hallan o establecen las definiciones sino el modo en que se utilizan; por ello, no es materia de examen el problema del origen de dichas definiciones.

${ }^{29}$ P. Moraux dice, en relación con DC I, que "Toute la démonstration de l'unicité se déroule in abstracto" (cf. Moraux, P.: "Introduction", en Aristote: Du ciel. Texte établit et traduit par P. Moraux. PUF. Paris. 1965, p. LXVIII). Claramente, alude a la ausencia de referencias históricas. Sin embargo, se puede contemplar que Aristóteles está utilizando sus presupuestos básicos, las definiciones, para elaborar tales demostraciones. 
(1) Def1 ${ }^{30}$ la naturaleza es principio del movimiento (268b16), o la

(2) Def2 la traslación es el movimiento con respecto al lugar (268b16), o

(3) Def3 el movimiento circular es el movimiento en torno al centro (268b21), o

(4) Def4 el movimiento rectilíneo es el movimiento ascendente o descendente (268b16-7), o

(5) Def5 el movimiento ascendente es el que se aleja del centro (268b22), o

(6) Def6 el movimiento descendente es el que se acerca al centro (268b23), o

(7) Def7 grave es lo que tiende naturalmente a desplazarse hacia el centro (269b23), y

(8) Def8 leve es lo que tiende naturalmente a alejarse del centro (269b24).

Estas definiciones constituyen una primera parte del soporte teórico necesario para la construcción de las demostraciones subsiguientes. Sin embargo, no lo agotan sino que luego, a medida que lo requiere el asunto, Aristóteles introduce nuevas definiciones.

\subsection{Prueba (i): hay éter (existe un cuerpo simple)}

A continuación citaremos un pasaje clave en donde Aristóteles prueba la existencia de un cuerpo simple que se corresponde con un movimiento natural también simple. El pasaje se encuentra en DC I 2, i. e., en el mismo inicio de la obra pero precisamente a posteriori de la introducción de la mayoría de las definiciones presentadas anteriormente. Parece claro que esta prueba constituye una de las más importantes del tratado no sólo por la ubicación en que se encuentra sino porque el hecho de asentar la existencia del éter es condición para poder luego pasar a probar sus propiedades (su

\footnotetext{
${ }^{30}$ Def para nombrar las definiciones. En cierto sentido, estas Def recogen tanto opiniones comunes como opiniones calificadas. La Def2 bien puede ser sostenida por cualquier individuo, mientras que las Def7 y 8 suponen una perspectiva teórica particular.
} 
finitud, su unicidad y su ingenerabilidad e incorruptibilidad), objeto de las pruebas que siguen a lo largo de DC I. Consideremos el pasaje en cuestión:

(i) Dado, pues, que existe el movimiento simple, que el movimiento circular es simple y que el movimiento del cuerpo simple es simple y el movimiento simple lo es de un cuerpo simple (en efecto, aun cuando lo fuera de uno compuesto, sería con arreglo al <elemento> predominante), es necesario que haya un cuerpo simple al que corresponda, de acuerdo con su propia naturaleza, desplazarse con movimiento circular (DC I 2 269a2-7) $)^{31}$

Reconstrucción de (i): si el movimiento simple (MS) conviene a un cuerpo simple (CS) y el movimiento circular (MC) conviene a un movimiento simple (MS), entonces el movimiento circular (MC) conviene a un cuerpo simple (CS) (D1 ${ }^{32} \mathrm{MSaCS}, \mathrm{MCaMS} \Rightarrow \mathrm{MCaCS}$ ). Existe, como se puede suponer, un desarrollo previo donde se inserta el pasaje, ${ }^{33}$ pero éste es el momento puntual donde se prueba la existencia del cuerpo simple que nos interesa resaltar ahora. En efecto, esta forma de reconstruir la prueba aristotélica bajo el formato del silogismo es un primer paso para la ulterior conexión con las definiciones expuestas en (2.1). Veamos las siguientes pruebas para poder desarrollar luego el modo en que podemos conectarlas en su conjunto con las definiciones mencionadas.

\subsection{Prueba (ii): el universo es finito}

El asunto discutido en DC I 5-6 consiste en la aporía referida a si el universo es finito o infinito en el espacio. En estos dos capítulos podemos encontrar, en resumen, unas dieciséis pruebas: unas más extensas y otras bien sintéticas. La mayor parte de ellas son reducciones a lo imposible

${ }^{31}$ Cf. Aristóteles, Acerca del cielo, Madrid, Gredos, 1996, Trad. Miguel Candel.

${ }^{32} \mathrm{D}_{\mathrm{n}}$ para referirme a las distintas deducciones que propondré. Las variables y su referencia al final del texto. "a" para caracterizar la relación entre los términos del juicio como universal afirmativa (a, i, e, o para los cuatro tipos de juicios según la caracterización estándar).

${ }^{33}$ Para un examen mucho más elaborado y puntilloso de la prueba de la existencia del éter o cuerpo dotado de movimiento circular, cf. Berrón, M., "Axiomatización, demostración y análisis en Acerca del Cielo", en Signos Filosóficos, UAM, Unidad Iztapalapa (México), Vol XIV, No 27 (enero-mayo de 2012), pp. 9-42. 
(adýnaton), i. e., demostraciones indirectas. Tomo una de las más interesantes, no casualmente la primera, que de algún modo también se repite en las pruebas subsiguientes. El pasaje dice así:

(ii) Pues, si lo que se desplaza en círculos fuera infinito, serían infinitos los $<$ radios $>$ trazados desde el centro. Y si éstos fueran infinitos, el intervalo también sería infinito. Llamo intervalo entre líneas a la magnitud en contacto entre líneas aparte de la que no es posible tomar ninguna otra. En efecto, éste será necesariamente infinito: pues los intervalos de radios limitados serán siempre limitados. Además, siempre es posible asumir a partir de lo dado algo mayor, así como llamamos al número infinito, que no tiene otro más grande, el mismo razonamiento $<$ hacemos $>$ acerca del intervalo; pues, si no es posible recorrer lo infinito, y si el cuerpo es infinito su intervalo será infinito, no se admitirá que se mueva en círculos: pero vemos el cielo girar en círculos. (....) (DC I 5 271b28-272a5; mi traducción)

En primer lugar, debemos notar que Aristóteles introduce una definición, la de intervalo, a mitad de la demostración en sentido estricto. Esto se condice con lo que señalamos antes referido a que Aristóteles introduce nuevas definiciones en la medida que lo requiere el asunto. Podemos afirmar entonces que tenemos una nueva Def, la Def9: intervalo es la distancia entre líneas (radios) (INT:=RA) (271b30-2). Consideremos, además, que se trata de una reducción a lo imposible ${ }^{34} \mathrm{y}$, en cuanto tal, el modo en que prueba es estableciendo una contradicción a partir de la asunción de la hipótesis contraria. ${ }^{35}$ En este caso, dado que lo que se quiere probar es que el universo

\footnotetext{
34 “Ahora bien, el razonamiento a través de lo imposible se demuestra cuando se pone la contradicción de la conclusión y se añade otra proposición, y se forma en todas las figuras" (APr. II 11 61a18-21.Trad. Candel).

35 Según el cuadro de oposición, una proposición universal afirmativa es contradictoria con una particular negativa; sin embargo, dado que la totalidad de los casos a los que nos referimos cuando hablamos sobre el universo está abarcada de por sí (en efecto, sólo hay un universo), a toda afirmación sobre el caso su negación será a la vez contraria y contradictoria.
} 
es finito, se asume que el universo es infinito. ${ }^{36}$ Reconstruimos tal afirmación como IaJ (infinito conviene a universo).

Reconstrucción de (ii): si infinito (INF) conviene a circular (CIR), y circular (CIR) conviene a radios (RA), entonces infinito (INF) conviene a radios (RA) (D2 INFaCIR, CIRaRA $\Rightarrow$ INFaRA); y si infinito (INF) conviene a radios (RA) y radios (RA) conviene a intervalo (INT), entonces infinito (INF) conviene a intervalo (INT) (D3 INFaRA, RAaINT $\Rightarrow$ INFaINT); ahora bien, si no recorrible (NR) conviene a infinito (INF) e infinito (INF) conviene a intervalo (INT), entonces no recorrible (NR) conviene a intervalo (INT) (D4 NRaINF, INFaINT $\Rightarrow$ NRaINT). Por otra parte, puesto que circular (CIR) conviene a radios (RA) y radios (RA) conviene a intervalo (INT), entonces circular (CIR) conviene a intervalos (INT) (D5 CIRaRA, RAaINT $\Rightarrow$ CIRaINT). Y circular (CIR) conviene a intervalo (INT) es convertible en intervalo (INT) conviene a circular (CIR) $($ CIRaINT $=$ INTaCIR $)$ y con la conclusión de D4 no recorrible $(\mathrm{NR})$ conviene a intervalo (INT) e intervalo (INT) conviene a circular (CIR), entonces no recorrible (NR) conviene a circular (CIR) (D6 NRaINT, INTaCIR $\Rightarrow$ NRaCIR). Pero esta conclusión es la que contradice la afirmación empírica "vemos el cielo girar en círculos" puesto que "no recorrible" significa que no se mueve, intransitable, mientras que circular se dice del cuerpo dotado de ese movimiento propio y éste es el cielo mismo. Así, la conclusión de D6, no recorrible (NR) conviene a circular (CIR) NRaCIR, se contradice con la afirmación recorrible ( $\neg \mathrm{NR})$ conviene a circular (CIR) $\neg \mathrm{NRaCIR}$ y éste es el absurdo.

\subsection{Prueba (iii $\mathrm{y}$ iv): el universo es único}

El problema abordado en los capítulos DC I 8-9 es si existe un único universo o si los universos son múltiples (DC I 8 276a17). ${ }^{37}$ Los siguientes

\footnotetext{
${ }^{36}$ Este enunciado es, en el universo aristotélico, un contrafáctico. En efecto, dado que en la óptica de Aristóteles el universo es finito, afirmar que el mismo es infinito es inadmisible. Además, a partir de tal enunciado se infieren afirmaciones que contradicen los fenómenos sensibles y ponen así en evidencia el carácter contrafáctico del enunciado.

${ }^{37}$ Para una reconstrucción más minuciosa de estos pasajes puede consultarse Berrón,
} 
pasajes ilustran dos pruebas que admiten su reconstrucción bajo el formato del silogismo. Las llamo (iii) y (iv) respectivamente. Cito los pasajes para su posterior reconstrucción:

(iii) Además, es forzoso que todos los mundos estén formados por los mismos cuerpos, al ser semejantes por naturaleza. Ahora bien, es forzoso también que cada uno de los cuerpos, v.g.: el fuego y la tierra y sus intermedios, tenga la misma potencia; pues si las cosas de allá sólo tienen en común el nombre con las que nos rodean y no se llaman así con arreglo a la misma forma, entonces también el mundo tendrá sólo el nombre tal. Es evidente, pues, que una de aquellas cosas tendrá por naturaleza que alejarse del centro y la otra acercarse al centro, si todo fuego es semejante al fuego y lo mismo cada uno de los demás elementos, como <ocurre con $>$ las partículas de fuego de este $<$ mundo $>$.

(iv) Que es necesario que ocurra así resulta evidente a partir de las hipótesis sobre los < distintos> movimientos: en efecto, los movimientos son limitados y cada uno de los elementos se define con arreglo a cada uno de los movimientos. De modo que, si los movimientos son los mismos, también los elementos serán necesariamente los mismos en todas partes. (DC I 8 276a30-b11, trad. de M. Candel).

Reconstrucción de (iii): si todo movimiento ascendente (MA) conviene a un movimiento rectilíneo (MR) y el movimiento rectilíneo (MR) conviene al fuego (FU), entonces el movimiento ascendente (MA) conviene al fuego (FU) (D7 MAaMR, MRaFU $\Rightarrow$ MAaFU); y si arriba (AR) conviene al final del movimiento ascendente (MA) y si el movimiento ascendente (MA) conviene al fuego (FU), entonces arriba (AR) conviene al fuego (FU) (D8 ARaMA, MAaFU $\Rightarrow$ ARaFU); y, como FU (nuestro fuego) es igual al fuego de allá (llamémoslo FU'), podemos seguir construyendo nuevas deducciones: si el movimiento rectilíneo (MR) conviene al fuego (FU) y el fuego (FU) conviene al fuego de allá (FU'), entonces el movimiento rectilíneo (MR) conviene al fuego de allá (FU') (D9 MRaFU, FUaFU' $\Rightarrow$ $\mathrm{MRaFU}$ ') y si movimiento ascendente (MA) conviene al movimiento rectilíneo (MR) y el movimiento rectilíneo (MR) conviene al fuego de allá (FU'),

M., "El soporte teórico subyacente en las pruebas de la unicidad del cielo en Acerca del cielo I 8 de Aristóteles", en Teorema, Universidad de Oviedo, 2015 (en prensa). 
entonces el movimiento ascendente (MA) conviene al fuego de allá (FU') (D10 MAaMR, MRaFU' $\Rightarrow$ MAaFU'). Por último: si arriba (AR) conviene al movimiento ascendente (MA) y el movimiento ascendente (MA) conviene al fuego de allá (FU'), entonces el arriba (AR) conviene al fuego de allá (FU') (D11 ARaMA, MAaFU' $\Rightarrow$ ARaFU'); y así hemos probado que el "fuego de allá" -y con idéntico razonamiento probaríamos lo mismo respecto de la tierra de allá y sobre los demás elementos- se dirige al mismo extremo superior -el "arriba"- que nuestro fuego. De este modo, encontramos que éste y aquél fuego, que son el mismo fuego, se dirigen al mismo y único lugar, el extremo superior de este único universo.

Reconstrucción de (iv): si limitado (LI) conviene a los movimientos (M) y los movimientos (M) convienen a los elementos (EL), entonces limitado (LI) conviene a los elementos (EL) (D12 LIaM, MaEL, $\Rightarrow$ LIaEL); y si límite (LI) conviene a movimiento (M) movimiento (M) conviene a "allá" (AL), entonces límite (LI) conviene a "allá" (AL) (D13 LIaM, MaAL $\Rightarrow$ LIaAL; y con ambas conclusiones, aunque invirtiendo el orden de los términos de la primera, ${ }^{38}$ probar lo siguiente: si elemento (EL) conviene a límite (LI) y límite (LI) conviene a "allá" (AL), entonces elemento (EL) conviene a "allá" (AL) (D14 ELaLI, LIaAL $\Rightarrow$ ELaAL; y con esta conclusión: si tierra $(\mathrm{T})$ conviene a movimiento $(\mathrm{M})$ y movimiento $(\mathrm{M})$ conviene a "allá" (AL), entonces tierra (T) conviene a "allá" (AL) (D15 TaM, MaAL $\Rightarrow$ TaAL). Y esta última conclusión, TaAL: tierra conviene a "allá", implica que aquél otro hipotético mundo cuenta con los mismos elementos que el nuestro, que los elementos de los dos hipotéticos mundos son los mismos y que o bien sus movimientos son antinaturales (lo que es imposible) o bien aquél y este mundo son uno y el mismo.

\subsection{Prueba (v): el universo es ingenerado e incorruptible}

En DC I 11 y 12 Aristóteles se ocupa de probar que el universo no tiene un origen ni tendrá un fin, i.e., que es ingenerado e incorruptible. Pero DC I 11 se ocupa en primer lugar de esclarecer qué significan estos términos

\footnotetext{
${ }^{38}$ Si bien la conversión de una universal afirmativa es en una particular afirmativa, en este caso, teniendo el conjunto completo de los elementos a los que conviene un límite, también se puede decir que el límite conviene a todo elemento de modo universal.
} 
y, para aclarar y precisar las distintas opciones que existen sobre ellos, selecciona como definiciones específicas las siguientes: incorruptible "en sentido más propio es lo que existe y que es imposible que se destruya de tal manera que, existiendo ahora, más adelante no exista o pueda no existir" (DC I 11 280b31-33) e ingenerado es "lo que es imposible o que no puede generarse de manera tal que primero no exista y luego sí" (DC I 11 281a4-6). En ambas definiciones Aristóteles sostiene que ser incorruptible o ingenerado depende de una imposibilidad (adýnaton), es decir, de una suerte de incapacidad o impotencia para realizar tal acción y, por ello, requiere aclarar qué entiende por adýnaton. La definición se halla un poco más adelante al definir "la potencia (dj́namis) en relación con el fin y lo máximo" (DC I 11 281a189). ${ }^{39}$ Esta definición persigue conectar la capacidad de acción de un ente respecto de un fin y un máximo posibles acordes con su naturaleza propia. Desde luego, Aristóteles asume que tener una potencia supone, en un cierto tiempo, la posibilidad de su realización. Por otra parte, conviene hacer la salvedad de que Aristóteles está discutiendo, a partir de DC I 10, con dos opiniones diversas y consideradas por él inviables: 1 . que el universo es engendrado pero incorruptible (Platón en el Timeo) y 2. que el universo es engendrado pero corruptible (atomistas y Heráclito). Veamos dos pasajes en donde se prueba la incorruptibilidad y la ingenerabilidad del universo:

(v) De modo que, si algo que existe durante un tiempo infinito es corruptible, tendrá la potencia de no existir. Y por ser durante un tiempo infinito, supóngase realizado lo que < puede llegar a ser>. En consecuencia, existirá y no existirá simultáneamente en acto. Se concluirá, pues, en una falsedad, dado que se ha establecido algo falso. Pero si no fuera algo imposible, tampoco la conclusión sería imposible. Por consiguiente, todo lo que existe siempre es incorruptible sin más. (DC I 12 281b20-25).

\footnotetext{
${ }^{39}$ Las dos primeras definiciones son casi tautológicas puesto que, en el caso de "incorruptible", incluye en el definiens el verbo cuya raíz contiene la "corruptibilidad" (phthartós, corruptible y phtharênai, para destruir). Lo que define propiamente es lo segundo: que no puede existir unas veces y otras no, i. e., existe siempre. Por ello, tenemos la Def10: lo incorruptible es lo que existe siempre (INC:=ETI); y por un razonamiento semejante, tenemos la Def11: lo ingenerado es lo que existe desde siempre (ING:=EDS). Finalmente, también tenemos la Def12: la potencia es la realización del fin y lo máximo (PO:=FM).
} 
Antes de reconstruir esta prueba, debemos considerar que, tal como hemos visto en la prueba (ii), nos encontramos también aquí con una reducción a lo imposible puesto que se arriba a una conclusión que es falsa y contraria con la que queremos probar. Por ello, las premisas de que se parte contienen suposiciones que se asumen contrarias a lo que se quiere probar. Veamos cómo es el razonamiento en la siguiente reconstrucción posible.

Reconstrucción de (v): si la corruptibilidad (CO) conviene a potencia (PO) y potencia (PO) conviene a existir en un tiempo infinito (ETI), entonces, la corruptibilidad (CO) conviene a existir en un tiempo infinito (ETI) $(\mathbf{D} 16 \mathrm{COaPO}$ y POaETI $\Rightarrow$ COaETI). Y si existir en un tiempo infinito (ETI) conviene a algo $(\mathrm{X})$ y la corruptibilidad (CO) conviene a existir en un tiempo infinito (ETI), entonces la corruptibilidad (CO) conviene a algo (X). (D17 ETIaX, COaETI, $\Rightarrow$ COaX).

Pero la conclusión de D17 supone admitir para una cosa X que existe y no existe en el mismo tiempo lo cual, evidentemente, es insostenible o, como dice Aristóteles, imposible. En efecto, la opinión platónica de que el universo es engendrado e incorruptible se vuelve absurda puesto que implica asumir, desde un punto de vista lógico, que algo puede ser y no ser al mismo tiempo. La prueba no parece ser convincente para quien creyera que el universo es simplemente corruptible, pero el destinatario de la refutación no es precisamente éste sino el platónico y, por ello, en dicho marco, la prueba alcanza su objetivo.

En un contexto semejante, tanto desde el punto de vista conceptual como de la confrontación con hipótesis rivales, Aristóteles elabora un argumento del mismo tenor que el (v) para mostrar que es imposible sostener la generabilidad para un universo eterno. Dice el pasaje:

(vi) Igualmente es ingenerable: pues si fuera generable, sería posible que durante un tiempo no existiera. (En efecto, es corruptible lo que, habiendo existido previamente, ahora no existe o puede que luego, en algún momento, no exista; generable, lo que puede no haber existido previamente.) Pero no hay ningún tiempo en que sea posible que lo que existe siempre no exista, ni < tiempo> infinito ni limitado: en efecto, si realmente existe durante un tiempo infinito, también puede existir durante un tiempo limitado. No cabe, por tanto, que una misma cosa pueda existir siempre y no existir nunca. ( $D C$ I 12 281b25-34). 
Reconstrucción de (vi): si generable (GE) conviene a potencia (PO) y potencia $(\mathrm{PO})$ conviene a existir en un tiempo infinito (ETI), entonces generable (GE) conviene a existir en un tiempo infinito (ETI) (D18 GEaPO y $\mathrm{POaETI} \Rightarrow \mathrm{GEaETI}$ ). Y si existir en un tiempo infinito (ETI) conviene a algo (X) y generable (GE) conviene a existir en un tiempo infinito (ETI), entonces generable (GE) conviene a algo (X). (D19 ETIaX, GEaETI, $\Rightarrow$ GEaX).

Nuevamente, la conclusión de D19 nos conduce a un absurdo que consiste en afirmar que algo que es eterno en el tiempo tiene también la potencia de no existir y, así, puede darse el caso en un tiempo infinito de que algo exista y no exista simultáneamente. Otra vez nos encontramos con el rechazo de la posición platónica de que un universo puede ser generado pero, a la vez, eterno. Aristóteles argumenta contra esta tesis a partir de sus presupuestos.

\section{Las pruebas y las definiciones}

Podemos tomar las definiciones presentadas en 2.1 e indagar acerca de su conexión con tales demostraciones. El interrogante que pretendemos responder es en qué medida es posible integrar las definiciones con las demostraciones de los tratados científicos. Desde luego, uno de los problemas a salvar es que las Def tienen que poder adaptarse al formato silogístico. Como hemos dicho, sostenemos que las Def funcionan como un soporte integral subyacente a todo DC I 8 y, por ello, más o menos explícitamente, están presentes a lo largo de todo el capítulo. Podemos encontrar integradas las Def con las pruebas reconstruidas del siguiente modo:

1) Un ejemplo concreto está en la premisa MAaMR (movimiento ascendente conviene a movimiento rectilíneo) de la D10 en la prueba (iii) puesto que ésta conecta al movimiento ascendente con el movimiento rectilíneo y la Def4 sostiene precisamente eso: que el movimiento rectilíneo es el movimiento ascendente o el descendente. Así, la Def4 da origen directamente a la premisa que contiene la definición: MR:=MA.

2) Otra opción interesante es vincular la prueba (iii) con la Def1: en primer lugar, tomamos la Def1 para obtener la premisa NA:=M y, entonces, merced a que el movimiento puede ser rectilíneo o circular (por la Def4), tenemos que MR=:NA (el movimiento rectilíneo es 
natural) a lo que podemos sumar que NAaFU (que la naturaleza -en tanto que principio del movimiento- se dice del fuego) y así obtenemos la conclusión MRaFU, que es la otra premisa utilizada en la D9 (D20 MR=:NA, NAaFU $\Rightarrow$ MRaFU). Más complejo puede ser el apoyo si consideramos la Def2: en efecto, podemos argumentar que la traslación (TRA) es el movimiento con respecto al lugar (LU), y dado que el movimiento con respecto al lugar (LU) conviene a la naturaleza (NA), entonces la traslación (TRA) conviene a la naturaleza (NA) (D21 TRA:=LU, LUaNA $\Rightarrow$ TRAaNA). Y con esta conclusión, considerando que rectilíneo (MR) conviene a traslación (TRA) y que traslación (TRA) conviene a naturaleza (NA), entonces rectilíneo (MR) conviene a naturaleza (NA) (D22 MRaTRA, TRAaNA $\Rightarrow$ MRaNA), que es la primera premisa de la $\mathbf{D} 20$.

3) Además, podemos encontrar un fundamento en las Def5 y Def6 para la prueba (iv): tomando como punto de partida la Def5, podemos deducir que si arriba (AR) es ascendente (MA) y ascendente (MA) conviene a elemento (EL), ${ }^{40}$ entonces arriba (AR) conviene a elemento (EL) (D23 AR=:MA, MAaEL $\Rightarrow$ ARaEL) y luego, con la nueva premisa movimiento $(\mathrm{M})$ conviene a arriba (AR) y la conclusión de $\mathbf{D} 23$, arriba (AR) conviene a elemento (EL), entonces movimiento (M) conviene a elemento (EL) (D24 MaAR, $\mathrm{ARaEL} \Rightarrow \mathrm{MaEL}$ ) que es una de las premisas iniciales de la prueba (iv) en la D12. Por otro lado, comenzando con la Def6, descendente (MD) es hacia el centro (CEN) y hacia el centro (CEN) conviene a "allá" (AL), entonces descendente (MD) conviene a "allá" (AL) (D25 $\mathrm{MD}:=\mathrm{CEN}, \mathrm{CENaAL} \Rightarrow \mathrm{MDaAL})$ y con esta conclusión descendente (MD) conviene a "allá" (AL) más la premisa movimiento (M) conviene a descendente (MD), podemos concluir que movimiento (M) conviene a "allá" (AL) (D26 MaMD, MDaAL $\Rightarrow$ MaAL), que es otra de las premisas iniciales utilizadas en la prueba (iii) en la D13.

4) Por otra parte, en el marco general de la prueba (ii) nos encontramos con la dificultad de que se trata, como se dijo, de una reducción

\footnotetext{
${ }^{40}$ En este punto en particular conviene hacer la salvedad de que no se está generalizando sobre el conjunto de todos los elementos sino, desde luego, sobre los elementos que tienen movimiento natural ascendente, particularmente, sobre el fuego. Aquí debería leerse "elemento con propiedad ascendente" (= fuego).
} 
a lo imposible. Por ello, algunas premisas son falsas (al menos son contrarias a la que se quiere defender y, por ello, a la que concuerda con las hipótesis o puntos de partida). En D2 tenemos la primera premisa -infinito (INF) conviene a circular (CIR), INFaCIR-como contradictoria a finito (limitado) (FI) conviene a circular (CIR) (FIaCIR). Esta premisa se puede deducir vía la Def3 del siguiente modo: puesto que finito (limitado) (FI) conviene al movimiento en torno al centro (CEN) y que el movimiento en torno al centro (CEN) conviene a circular (CIR), entonces finito (limitado) (FI) conviene a circular (CIR) (D27 FIaCEN, CEN=:CIR $\Rightarrow$ FIaCIR). De este modo, con la $\mathbf{D} 27$ podemos tener un apoyo en la Def3 para la contraria de la primera premisa de la $\mathbf{D} 2$.

5) Una opción llana para ver cómo se introducen las definiciones en la demostración (ii) está en el uso explícito que hace Aristóteles de la definición de intervalo en la prueba misma. En efecto, al definir según Def9 al intervalo como la distancia entre las líneas (los radios en este caso) se está dando el fundamento para las siguientes premisas: en D3, para la premisa RAaINT y en $\mathbf{D 5}$ para la premisa RAaINT.

6) También a partir de la Def3 se puede deducir la primera premisa de la D1: si el movimiento circular (MC) es el movimiento en torno al centro (CEN) y el movimiento en torno al centro (CEN) conviene a un movimiento simple (MS), entonces el movimiento circular (MC) conviene a un movimiento simple (MS), (D28 MC:=CEN, CENaMS $\Rightarrow$ MCaMS).

7) En lo referido a la prueba (v) cuyo objeto es probar la incorruptibilidad del cosmos y recordando que se trata de una prueba por reducción a lo imposible, podemos ver que la conclusión de D16 es contraria a la Def10. En efecto, la conclusión es que la corruptibilidad (CO) conviene a existir en un tiempo infinito (ETI) (COaETI) que claramente contradice la Def10: lo incorruptible es lo que existe siempre (INC:=ETI).

8) En el caso de la prueba (iv) referida a la ingenerabilidad del universo, podemos reconstruir la siguiente deducción a partir de la Def11 para llegar a la contraria de la conclusión de D19 (siempre recordando que también en esta prueba tenemos una reducción a lo imposible). Si ingenerable (ING) es existir desde siempre (EDS) y 
existir desde siempre (EDS) conviene a existir en un tiempo infinito (ETI), entonces ingenerable (ING) conviene a existir en un tiempo infinito (ETI) D29 ING:=EDS y EDSaETI $\Rightarrow$ INGaETI). Y con esta conclusión de $\mathbf{D 2 9}$ podemos también deducir que si ingenerable (ING) conviene a existir en un tiempo infinito (ETI) y existir en un tiempo infinito (ETI) conviene a algo (X) (la P1 de D19), entonces ingenerable (ING) conviene a algo (X) (D30 INGaETI y ETIaX $\Rightarrow$ INGaX). Esta conclusión es también contraria a la conclusión de D19 que sostiene que generable (GE) conviene a algo $(\mathrm{X}) \mathrm{y}$, de este modo, tenemos una nueva contradicción para la reducción al absurdo.

Luego de este trabajo de reconstrucción de pruebas y de conexión de las pruebas con las definiciones bajo el formato del silogismo, pasemos a considerar aspectos generales concernientes a algunas consecuencias epistemológicas de dichas reconstrucciones.

\section{Definiciones y conclusiones, lo que podemos esperar de una axio- matización de DC I}

Una presentación pedagógica estándar de aquello que debemos esperar de una ciencia axiomatizada puede ser caracterizada del siguiente modo: en un sistema axiomático tenemos un conjunto reducido de principios o axiomas a partir de los que se infieren -según una lógica específica y, en el caso de Aristóteles, la del silogismo- un conjunto significativamente mayor de proposiciones. Al conjunto menor de proposiciones se las denomina "básicas" y al conjunto mayor "derivadas". Esta somera caracterización se atribuye de modo estándar a Aristóteles particularmente a partir de Scholz ${ }^{41}$ y de Barnes, ${ }^{42}$ pero desde entonces también en la gran mayoría de las interpretaciones vigentes. ${ }^{43}$ Sin embargo, si observamos el conjunto de

\footnotetext{
${ }^{41} \mathrm{El}$ artículo de Scholz es de 1930, pero fue reeditado en Scholz, H., "The Ancient Axiomatic Theory", en Barnes, J. M. Schofield y R. Sorabji, (Ed.) (1975): Articles on Aristotle, Vol. 1, Science, London, Duckworth, 1975.

${ }^{42}$ Barnes, J., op. cit.

${ }^{43}$ Lecturas más recientes en la misma dirección: Hintikka, J., "On the Ingredients of an Aristotelian Science”, en Nous VI - 1, 1972; Vega Reñón, L., La trama de la demostración, Madrid, Alianza Universidad, 1990, y Cassini, A., op. cit..
} 
las demostraciones presentadas anteriormente podemos constatar que las cosas no se presentan según esta visión estándar. Por el contrario, podemos observar varios aspectos del asunto que se presentan de modo particular. Veamos algunos de ellos:

(1) Un repaso del conjunto de las deducciones D1-D30 nos muestra que contamos con cuatro conclusiones generales (los objetos de demostración); doce definiciones; treinta y nueve términos; y treinta deducciones.

(2) Es sumamente significativo que para probar sólo cuatro proposiciones se requiera de semejante estructura argumentativa. El nivel de persuasión aumenta si tenemos en cuenta lo poco que ha sido reconstruido bajo el formato del silogismo; en efecto, si realizáramos tal labor con mayor base textual, las deducciones se multiplicarían y con ellas los términos e, incluso, encontraríamos nuevas definiciones presentadas en otros lugares del tratado. Debemos tener en cuenta, por ejemplo, que la prueba (ii) es la primera de dieciséis (que también podrían ser reconstruidas y a las que podríamos encontrarles su conexión con las definiciones en cuestión). Además, todas estas pruebas apuntan a una y la misma conclusión. Las pruebas (iii) y (iv) son también las primeras de siete y cabe para ellas idéntico razonamiento. Todas estas reconstrucciones, las realizadas y las realizables, ponen en evidencia la poderosa trama argumentativa que coadyuva en las pruebas de los cuatro tópicos abordados. Una lectura como la que hemos realizado de $D C$ I nos permite hacer explícito este trasfondo teórico que de otro modo puede pasar relativamente inadvertido. La tesis de Gotthelf sobre una trama argumentativa subyacente para Partes de los animales tiene en $D C$ I un fuerte correlato, quizá más explícito aún. Ciertamente, observamos que las definiciones presentadas en un comienzo de $D C$ aumentan en número según lo requiera el asunto; pero lo más significativo es que continúan operando a lo largo de todo DC I. Esta peculiaridad muestra claramente el plan de Aristóteles a la hora de la redacción del libro: exponer las hipótesis centrales -las definiciones- que le permitan probar uno de los puntos centrales de su cosmología: la existencia de un cuerpo simple dotado de movimiento circular simple. Una vez realizado esto, procede a probar sus atributos fundamentales y, para ello, reutiliza las definiciones aumentándolas según la necesidad de la ocasión. Su plan consiste en postular las definiciones necesarias para la primera demostración y luego aumentar el soporte teórico en la medida que lo requieran los nuevos desafíos. 
(3) Además, es relevante que en tal estructura argumentativa se encuentren las doce definiciones aludidas por otro detalle epistemológico vinculado al carácter específico que deben tener las demostraciones. Recordemos que la forma -el silogismo- no es condición suficiente sino necesaria ${ }^{44}$ para que haya una genuina apódeixis científica. Al soporte formal dado por el rigor del silogismo debemos sumarle las definiciones operando como premisas de los razonamientos. ${ }^{45}$ Este detalle es determinante porque de no contar con dichas definiciones como soporte teórico de las deducciones, no tendríamos verdaderas demostraciones.

(4) Otro aspecto relevante es la conexión que se establece con el rendimiento explicativo de las definiciones. La interpretación de Charles comentada en (1.b) que traba a la definición con la demostración tiene aquí un buen caso de prueba. Si su interpretación es correcta, deberíamos obtener certeza de que las definiciones utilizadas son genuinas definiciones sí y sólo sí ellas actúan como soporte de demostraciones científicas. Recordemos que Charles le otorga al método de la demostración un valor preponderante puesto que tal método permite satisfacer las -por él llamadas- restricciones de simplicidad y definicional. Más explícitamente, afirma que nuestra práctica de la definición se apoya en nuestra práctica de la demostración. ${ }^{46}$ Esta primera asociación entre las dos prácticas supone, luego, una nueva conexión extremadamente relevante puesto que, de ser correcto el enfoque, existiría una co-determinación entre la esencia (la definición) y la causali-

\footnotetext{
44 "Necesaria" pero en un sentido - si se admite- débil. Recordemos la tesis de Gotthelf según la cual, para que haya una demostración, basta con que las demostraciones soporten el formato del silogismo. "Necesaria" en un sentido fuerte expulsaría del universo de las demostraciones a todo argumento no expresado según esta forma.

${ }^{45}$ Es la diferencia presentada en Top. I 1 sobre los tipos de razonamientos: los razonamientos dialécticos partes de opiniones reputadas (éndoxa) mientras que los razonamientos demostrativos parten de premisas verdaderas y primordiales (Top. I 1 100b1-2); pero todos son razonamientos. En consonancia con la nota precedente, si el formato del silogismo fuera requerido explícitamente para las demostraciones científicas, ¿por qué no requerirlo también para los razonamientos dialécticos? Curiosamente, si entendemos esta exigencia en un sentido fuerte, en línea con la tesis de Barnes, ni habría demostración ni habría razonamiento dialéctico.

${ }^{46}$ Charles, D., op. cit. p. 204.
} 
dad. ${ }^{47}$ Además, podemos tener certeza de que las definiciones utilizadas son verdaderas definiciones científicas dado que fuimos capaces de utilizarlas como premisas explicativas de los distintos tópicos examinados. Las definiciones brindan por medio de sus términos el "término medio" que sirve de causa en la demostración.

(5) También es importante otra peculiaridad relacionada con el número de definiciones utilizadas como principios en las múltiples demostraciones. En efecto, es notorio que las definiciones actúan en conjunto en la prueba de los distintos puntos en discusión. Esto atenta directamente contra la visión estándar de la axiomatización puesto que en ésta pocos principios apuntalan múltiples conclusiones mientras que aquí, inversamente, encontramos que una conclusión se vale de muchos principios. ¿Cómo es posible esta pluralidad? Debemos recordar que las distintas definiciones ofrecen contenido explicativo que apela a aspectos distintos del fenómenos: la Def1 refiere a la naturaleza en general como principio del movimiento mientras que, por caso, las Def7 y Def8 aluden a la condición de grave o leve de todo elemento. Son aspectos que coexisten en los cuerpos simples y, por ello, pueden actuar como causas, bajo condiciones distintas y en sentidos distintos, del mismo fenómeno. Pero esto también es posible porque la presencia de las distintas definiciones para las mismas conclusiones se da en distintas formas de llevar a cabo un análisis. Esto nos conduce al siguiente punto.

(6) De lo anterior se sigue que cada demostración es una forma de realizar el análisis (según lo caracteriza Detel ${ }^{48}$ ), i.e., de realizar una construcción de deducciones que, al integrar elementos definicionales con premisas de otro orden (como en la prueba (ii), donde hay premisas de contenido empírico), permiten probar la conclusión en cuestión. Cada demostración, cada análisis, incluye premisas distintas y definiciones distintas (de ahí su divergencia) y, por ello, cada una es valiosa en sí misma. De este modo, todas en conjunto constituyen un frondoso soporte teórico que vuelve más fundada, compleja y rica a la conclusión y, asimismo y por ello, más evidente.

(7) Por otra parte, otra inferencia puede ser extraída del desarrollo de (2) y (3): estas consideraciones nos permiten dimensionar que el aparato

47 Ibid.: p. 217.

${ }^{48}$ Tanto en Detel, W., "How all animals have stomach", como en "Aristotle's Logic and Theory of Science". 
argumentativo desarrollado por Aristóteles, al menos en su faz demostrativa, se articula en una poderosa red arborescente en cuyos extremos es factible hallar las definiciones. Pero en la medida en que las demostraciones se multiplican, la red se vuelve más compleja y precisamente en esa complejidad es dable estimar que Aristóteles encuentra el auténtico carácter persuasivo de las demostraciones. Éstas, agrupadas y expuestas unas tras otras constituyen un todo que conjuntamente adquiere una, cada vez, mayor convicción (pistis).

(8) Finalmente y a la luz de estos corolarios, hemos encontrado una forma alternativa para una axiomatización posible en Acerca del cielo de Aristóteles. Tenemos unas pocas proposiciones que son la conclusión de una multiplicidad de premisas. Entre estas premisas encontramos definiciones, i. e., principios, que son más numerosos que las conclusiones.

\section{Consideraciones finales}

En la introducción de este trabajo anunciamos nuestros propósitos y el marco para su desarrollo. Por un lado, explicitamos nuestra filiación a una opción "silogicista moderada" que supone que la lógica implícita de las demostraciones científicas es la del silogismo. Además, resulta importante señalar el modo en que distintos trabajos mencionados en (1) tales como los de Gotthelf, Detel y Charles, llegan a coincidir ${ }^{49}$ en algunos puntos específicos y relevantes: 1) la posibilidad de reconstruir argumentos en los textos de ciencia natural; y 2) el uso de las definiciones como principios demostrativos y explicativos en los tratados de ciencia natural. También abordamos, aunque someramente, la conexión entre la definición y la demostración. Sin embargo, nuestro objetivo central consistió en examinar el modo en que las definiciones se insertan en la trama argumentativa en la medida en que ellas son consideradas como los principios de las ciencias particulares. En efecto, el complejo tópico de la relación entre la definición y la demostración -que he abordado sólo de soslayo- es de una profundidad que amerita un examen más extenso; aquí sólo fue considerado en virtud de mostrar su conexión con el conjunto de la trama argumentativa.

${ }^{49}$ Naturalmente, no haciendo hincapié en las diferencias. 
En virtud de que nuestro propósito apuntaba a constatar el uso de un mismo soporte teórico para todas las demostraciones de DC I llevamos a cabo en (2) la reconstrucción de un conjunto de pruebas extraídas de distintos capítulos de dicho libro I. A su vez, en conexión con esta selección de pruebas, buscamos mostrar en (3) que ellas utilizan un mismo soporte teórico constituido por una serie de definiciones presentadas al comienzo del tratado que se amplia en la medida que lo requiere la prueba en cuestión. El desarrollo de (3), y luego de (4), persiguió mostrar que tal soporte teórico opera permanentemente en $D C$ I. Destacamos también que esta conexión entre las prueba y su soporte teórico fue realizada por medio de reconstrucciones en formato silogístico. Una vez vuelto explícito esto, pudimos llevar a cabo una nueva valoración, pero esta vez en la relación que existe entre el conjunto de premisas y el de las conclusiones. En efecto, logramos volver evidente que para sólo cuatro proposiciones que buscan ser probadas, se requiere un número importante de definiciones pero también un número importante de otras premisas. Hecha esta constatación, se obtiene una particular forma de axiomatización en donde llegan a ser más las definiciones o principios que las conclusiones o teoremas. Resulta característico de esta forma de reconstruir las pruebas un resultado alternativo al estándar puesto que, precisamente, no se cumple con el requisito ordinario para cualquier axiomatización según el cual los principios son mucho menores que las conclusiones. En este sentido, podemos entender de un modo sugerentemente diverso el modo en que puede presentarse una ciencia axiomatizada en clave aristotélica. 


\section{Diccionario de Variables:}

\begin{tabular}{|l|l|l|l|}
\hline AL & "allá" & INT & intervalo \\
\hline AR & Arriba & LE & Leve \\
\hline CEN & Centro & LI & Limitado \\
\hline CIR & Circular & LU & Lugar \\
\hline CO & Corruptibilidad & M & Movimiento \\
\hline CS & Cuerpo simple & MA & Movimiento ascendente \\
\hline EDS & Existe desde siempre & MC & Movimiento circular \\
\hline EL & Elemento & MD & Descendente \\
\hline ETI & $\begin{array}{l}\text { Existencia en un tiempo } \\
\text { infinito o existir siempre }\end{array}$ & MR & Movimiento rectilíneo \\
\hline EX & Extremo & MS & Movimiento simple \\
\hline FI & Finito & NA & Naturaleza \\
\hline FM & Fin y máximo & NR & No recorrible \\
\hline FU & Fuego & PO & Potencia \\
\hline FU' & Fuego "de allá" & RA & Radio \\
\hline GE & Generable & T & Tierra \\
\hline GRA & Grave & TR & Tres \\
\hline IMED & Intermedio & TRA & Traslación \\
\hline INC & Incorruptible & U & Universo \\
\hline INF & Infinito & $\mathrm{X}$ & Algo \\
\hline ING & Ingenerado & & \\
\hline & & & \\
\hline
\end{tabular}

Recibido 8/2014; aceptado: 11/2014 